\title{
SER Y ESTAR SON PAN COMIDO: LA ENSEÑANZA DE LA GRAMÁTICA MEDIANTE EXPRESIONES IDIOMÁTICAS
}

\author{
Laura Marqués Pascual \\ Stefan DuBois
}

\section{(ब) $\Theta \Theta$}

Esta obra está bajo una licencia Creative Commons 



\title{
SER Y ESTAR SON PAN COMIDO: LA ENSEÑANZA DE LA GRAMÁTICA MEDIANTE EXPRESIONES IDIOMÁTICAS
}

\author{
SER AND ESTAR ARE PIECE OF CAKE: TEACHING GRAMMAR \\ WITH IDIOMATIC EXPRESSIONS
}

\author{
Laura Marqués Pascual \\ Stefan DuBois
}

\begin{abstract}
RESUMEN
Este artículo presenta un estudio sobre la enseñanza de expresiones idiomáticas, modismos o frases hechas como ser un cero a la izquierda en un curso de español como lengua extranjera de nivel intermedio. Tomando como punto de partida recomendaciones de incorporar las expresiones idiomáticas en la enseñanza de clases de segunda lengua (ej. Irujo, 1986b; Liontas, 2003; Tran, 2012), el estudio investiga un acercamiento pedagógico que hasta ahora no ha recibido mucha atención: el uso de los modismos como recurso mnemónico en el aprendizaje de aspectos gramaticales de la lengua. Se aprovecharon los materiales didácticos de Zyzik (2010) y Zyzik y Marqués-Pascual (en prensa) para comparar dos métodos diferentes de la enseñanza explícita de la distinción semántica entre ser y estar: uno con actividades de reconocimiento y producción y otro mediante la enseñanza de expresiones idiomáticas. Los resultados indicaron que la enseñanza de modismos no es necesariamente mejor que otros métodos de enseñanza, pero sí es una opción viable para la práctica de la gramática a la vez que fomenta el aprendizaje del lenguaje idiomático. Palabras clave: expresiones idiomáticas, modismos, ser y estar, acercamientos pedagógicos, español como segunda lengua.
\end{abstract}

\begin{abstract}
This article describes a study on the teaching of idiomatic expressions, idioms, or figures of speech such as ser un cero a la izquierda in an intermediate-level Spanish foreign language class. Using as a point of departure the recommendations of various authors (Irujo, 1986b; Liontas, 2003; Tran, 2012) to incorporate idiomatic expressions into foreign language classes, this study investigates a pedagogic approach which until now has not received much attention: the use of idioms as a mnemonic resource in learning grammar. With this in mind, didactic materials were prepared following those used by Zyzik (2010) and Zyzik \& Marqués-Pascual (in press) for the explicit teaching of the semantic distinction between ser and estar. Two pedagogic approaches were compared using these materials: one that presented the semantic distinction and was followed by input/output activities and the other via teaching idiomatic expressions. The results indicated that the instruction of idioms is not necessarily better than other teaching methods, but it certainly is a viable way to practice grammar while simultaneously exposing students to idiomatic language.

Key words: idiomatic expressions, idioms, ser and estar, pedagogic approaches, Spanish as a second language.
\end{abstract}

Dra. Laura Marqués Pascual. Universidad de California, Santa Bárbara. Departamento de Español y Portugués. Estados Unidos.

Correo electrónico: 1marques@spanport.ucsb.edu

Lic. Stefan DuBois. Universidad de California, Santa Bárbara. Departamento de Español y Portugués. Estados Unidos.

Correo electrónico: sdubois@umail.ucsb.edu 


\section{Introducción}

En los últimos años, se han publicado numerosos artículos que enfatizan tanto las virtudes como la necesidad de enseñar el lenguaje idiomático en el aula. Estos artículos destacan que la enseñanza de expresiones idiomáticas (como meter la pata o estar entre la espada y la pared) a menudo recibe atención insuficiente o poco adecuada en clases de segunda lengua (L2), tanto de inglés (Cooper, 1999; Liontas, 1999; Wray, 2000; Irujo, 1986b) como de español (Sugano, 1981; Liontas, 2003). Por ello, a pesar de la ubicuidad del lenguaje idiomático y fraseológico, ${ }^{1}$ los estudiantes de segunda lengua frecuentemente se encuentran poco preparados cuando se enfrentan con este tipo de unidades léxicas prefabricadas, ya que su significado es poco transparente la mayoría de las veces. Por ejemplo, Tran (2012) menciona cómo estudiantes vietnamitas que habían estudiado inglés como segunda lengua durante más de 6 años seguían siendo incapaces de comunicarse de manera eficaz debido a esta falta de competencia idiomática. Así, todos los estudios mencionados en el presente trabajo concluyen que el lenguaje idiomático debe recibir más atención en el aula de segunda lengua y proponen planes para clases específicamente dedicados a su enseñanza. Sin embargo, los maestros frecuentemente deben seguir programas de estudios bastante apretados o previamente establecidos y quizá no siempre sea posible encontrar tiempo para clases completamente dedicadas a la enseñanza del lenguaje idiomático o coloquial. De hecho, algunos autores como Zyzik (2011) y Cornell (1999) han reconocido que tal vez incluir el lenguaje idiomático en el aula de manera más orgánica tenga ciertas ventajas y han propuesto metodologías para su incorporación

Tomando como punto de partida estas recomendaciones, el estudio aquí descrito investigó un acercamiento pedagógico que hasta la fecha no había recibido mucha atención: el uso de expresiones idiomáticas, modismos o frases hechas (como ser un cero a la izquierda) en un curso de español como lengua extranjera como recurso facilitador en el aprendizaje de aspectos gramaticales de la lengua. El estudio se realizó con estudiantes de español como segunda lengua de nivel intermedio que recibieron instrucción explícita sobre los verbos copulativos ser y estar, con y sin la incorporación de modismos en el plan de clase. La mejor manera de presentar de manera explícita ser y estar o de incorporar la instrucción de modismos en el aula (como han hecho otros estudios, véanse Irujo, 1986a, 1986b; Liontas, 2003; o Zyzik, 2010) no fueron el objetivo de este estudio, sino el papel facilitador que puede tener la enseñanza de modismos en el aprendizaje de la gramática de una segunda lengua. El aprendizaje de ser y estar se consideró un tema ideal de estudio, en primer lugar, porque este concepto tiende a ser uno de los grandes desafíos del español para hablantes nativos de inglés (los cuales expresan los dos verbos copulativos con el único verbo "to be"), y en segundo lugar, por la facilidad y frecuencia con que estos verbos aparecen en combinaciones idiomáticas (poner a alguien entre la espada y la pared da como resultado estar entre la espada y la pared).

\section{Los modismos o expresiones idiomáticas}

Definir exactamente qué es un modismo no es una tarea fácil y son varios los autores que han tratado de llegar a una definición precisa. En términos generales, la mayoría concluye que el concepto de modismo es sobre todo un concepto borroso; una definición exacta frecuentemente se solapa con la de categorías similares (dichos, proverbios, locuciones, etc.) e inevitablemente excluye algún ejemplo que el sentido común dicta que tendría que incluirse. 
Como punto de partida, Zyzik (2010) cita la definición del Diccionario de la Real Academia de la Lengua: "una expresión fija, privativa de la lengua, cuyo significado no se deduce de las palabras que la forman". Así, la mayoría de los estudios previos concuerdan en que los modismos se caracterizan por dos rasgos sobresalientes de los demás: la idiomaticidad y la fijación.

En primer lugar, el significado de un modismo no se puede derivar intuitivamente de sus elementos constitutivos. Expresiones como mandar a alguien a freir espárragos, estirar la pata o tener algo entre ceja y ceja comportan un significado que va más allá del que se pueda deducir inmediatamente desde un nivel literal. Tampoco tienen un significado que se pueda deducir intuitivamente de la suma de sus componentes. Aunque Boers, Eyckmans y Stengers (2007) señalan que en teoría se puede conjeturar sobre el significado del modismo si se conoce la metáfora de la que proviene, para la mayoría de los hablantes los modismos resultan ser semánticamente opacos a primera vista debido a esta característica de idiomaticidad. En segundo lugar, los modismos son expresiones fijas ya que suelen exhibir una cierta rigidez en el nivel léxico y sintáctico y a menudo pierden su sentido figurativo si la forma establecida se desecha. Cooper (1998) provee el modismo en inglés John kicked the bucket (que podríamos traducir informalmente como "Juan estiró la pata") como un ejemplo que es sintácticamente inflexible - el sentido metafórico se pierde si se cambia la frase a la voz pasiva: The bucket was kicked by John sólo connota un sentido literal (p. 257).

No obstante, los dos criterios de idiomaticidad y fijación son tendencias más que reglas absolutas. Cooper señala que algunos modismos exhiben una cierta transparencia semántica: la decepción o insulto implicado por un slap in the face (que hasta se podría traducir literalmente como "una bofetada en la cara") no queda muy alejado del significado literal y físico. Del mismo modo, no todos los modismos exhiben una estructura fija inamovible, y aunque la mayoría de modismos son estructuras completamente fijas, otros presentan variabilidad sintáctica. Mientras que el ejemplo anterior de to kick the bucket pierde el sentido figurativo al cambiarlo a la voz pasiva, Cooper ofrece las frases "We rolled the red carpet out for the King" y "The red carpet was rolled out for the King" como ejemplos de un modismo más flexible.

A pesar de las imperfecciones que se reconocen en la definición expuesta, el presente estudio sigue estas características mencionadas. Cabe destacar también que, en cuanto a qué término usar para referirnos al lenguaje idiomático que nos interesa, en el presente trabajo se sigue la recomendación de Zyzik (2010) y se usa el término modismo, aunque existe una gran variedad de palabras que se refieren a conceptos similares (locuciones, frases hechas, expresiones fijas, etc.).

\section{La enseñanza de modismos en la clase de L2}

El reconocimiento de la necesidad de incluir la enseñanza activa de modismos en el aula ha dado origen a varias propuestas pedagógicas. Algunos autores como Cooper (1999) y Cornell (1999) admiten que el número de modismos en una lengua es demasiado grande como para esperar que los estudiantes memoricen una lista exhaustiva, pero al mismo tiempo argumentan que ignorarlos completamente sería irresponsable por parte de los maestros de segunda lengua. Así, abogan por una solución intermedia en la cual se enseña a los estudiantes estrategias para enfrentar los modismos cuando surja la necesidad comunicativa de usarlos o procesarlos.

Uno de estos acercamientos es el propuesto por Liontas (1999) en un proceso de cuatro fases. Aunque por razones de espacio no es posible la descripción completa de los particulares 
de esta metodología, se comienza por presentar a los estudiantes un texto, un video, o un segmento de audio que contenga lenguaje idiomático en su L1 para hacer conscientes a los estudiantes de ejemplos de lenguaje idiomático en la lengua nativa. Después, se recomienda una variedad de pasos que exigen que los estudiantes usen pistas contextuales para adivinar el significado de modismos de la L2. Con este método Liontas enfatiza el uso, siempre que sea posible, de lenguaje auténtico en vez de artificial, el estudio de sus orígenes etimológicos, y no pasar demasiado tiempo con la definición de modismos en un texto a costa del significado global. Al explicar su metodología, Liontas reitera una y otra vez la importancia de la variedad en la presentación de los modismos y aconseja que el profesor siempre esté abierto a nuevas maneras de aumentar el interés de los estudiantes. Irujo (1986b) también enfatiza la variedad, e incluye en su propuesta actividades lúdicas. Los juegos ofrecen un recurso útil para los profesores por su naturaleza repetitiva, así como por su capacidad de transformar el aprendizaje en un proceso que anima a los estudiantes a disfrutar. De la misma manera, Cooper (1998) da una lista de actividades, incluyendo sugerencias como juegos de mesa y dibujos animados.

Todos estos autores sugieren clases dedicadas única y exclusivamente a la enseñanza de modismos. Este acercamiento difiere del propuesto por el presente estudio; mientras que dichos autores sugieren métodos para la enseñanza y la retención de modismos, nuestro estudio utilizará los modismos como un recurso pedagógico para promover o favorecer la enseñanza de conceptos gramaticales. También se verá hasta qué grado los estudiantes retienen los modismos que se utilizan siguiendo tal acercamiento.

Los diferentes estudios del impacto que puede tener la enseñanza de modismos en la adquisición de una L2 se han centrado sobre todo en diferentes maneras de enseñar los modismos y cómo facilitar su comprensión y aprendizaje. Por ejemplo, la importancia de la información contextual se ha explorado en varios estudios. Así, tanto Liontas (2003) como Irujo (1986b), quienes realizaron estudios con aprendices de español como lengua extranjera, demostraron que el contexto es sumamente importante para la comprensión de los modismos. También demostraron que cuanto más se parecen los modismos a su equivalente en la L1, más fácil es su interpretación, y que los aprendices pueden utilizar su conocimiento de modismos en su L1 en la comprensión de modismos en la L2.

Otros estudios dignos de mención son los realizados por Boers y sus colaboradores (2000, 2007, 2014), los cuales demostraron los beneficios mnemotécnicos de conocer el origen de una expresión a la hora de intentar reconocer un modismo (un proceso llamado etymological elaboration en inglés). Por ejemplo, Boers (2000) demostró que el conocimiento de la metáfora que existe detrás de un modismo facilita su retención. Boers, Eyckmans y Stengers (2007) también corroboraron los efectos mnemotécnicos de saber el origen de un modismo y, en menor grado, que este conocimiento del modismo le facilita al aprendiz la tarea de reconocer los contextos pragmáticos más adecuados para su uso. Más recientemente, el enfoque de esta línea de investigación se ha centrado en los efectos de la aliteración, y ha salido a la luz que esta cualidad hace que unas expresiones sean más fáciles de memorizar que otras (Linstromberg y Boers, 2008; Boers, Lindstromberg y Webb, 2014).

Otras maneras de enseñar los modismos en la clase son las propuestas por Zyzik (2010). En su estudio, llevado a cabo con estudiantes de español como lengua extranjera, se compararon dos métodos diferentes de presentar los modismos en el aula: una organización temática (por ejemplo, mala conducta o relaciones de interés) y una organización formal en cuanto al verbo principal del modismo (dar la lata, dar gato por liebre, dar la vuelta a la 
tortilla). Los resultados del estudio concluyeron que aunque la organización de los modismos (al menos la utilizada en el estudio) no afectó de manera significativa el aprendizaje, ambos grupos aprovecharon la instrucción que se les dio, aunque el nuevo conocimiento se reflejara más en el plano receptivo. Aunque estudios previos como los de Boers y sus colaboradores han abogado por la organización temática de modismos (desde una perspectiva más cognitiva), el estudio de Zyzik confirmó que una organización formal de los modismos es una opción perfectamente viable para la enseñanza y tal vez más práctica para el docente.

Todos los estudios mencionados tratan sobre la comprensión, el aprendizaje y la retención de modismos; sin embargo, hasta la fecha no parece que haya ningún estudio que específicamente investigue el uso de modismos como herramienta para alcanzar otras metas pedagógicas. El estudio que más se aproxima a este objetivo es el realizado por Zyzik y Marqués-Pascual (2012). Este estudio comparó dos tratamientos diferentes para la enseñanza de la "a" personal. Un tratamiento consistió en la enseñanza explícita de reglas acompañada de actividades de reconocimiento en contexto y posterior producción (input/output). El otro tratamiento consistió en la enseñanza de modismos que contenían numerosos ejemplos de la "a" personal (ej. dejar plantado a alguien), pero sin enseñanza explícita de las reglas que rigen el uso de la marca de objeto directo personal. El grupo de estudiantes que recibió enseñanza deductiva de las reglas seguida de actividades de input/output obtuvo mejores resultados en las pruebas de medición, dando a entender que este tratamiento fue más eficaz para el aprendizaje de la "a" personal. Sin embargo, un resultado revelador de este estudio fue que el grupo que recibió la enseñanza de modismos sin presentación de las reglas en cuanto al uso de la "a" personal también mejoró en las pruebas de evaluación en cuanto al uso de esta estructura. Las autoras proponen que la exposición a numerosos ejemplos de modismos con la "a" personal y su posterior uso en las actividades (como María pilló a Juan con las manos en la masa) promueven un aprendizaje incidental de esta estructura. Así, aunque el tratamiento con modismos no fue tan eficaz para el aprendizaje de la "a" personal como para el tratamiento de enseñanza explícita, el grupo obtuvo un doble beneficio, puesto que también aprendieron una serie de modismos que antes les eran desconocidos.

El presente estudio sigue la línea de investigación abierta por Zyzik y MarquésPascual (2012). En dicho estudio, el grupo que recibió enseñanza explícita de las reglas obtuvo mejores resultados en las pruebas de medición, y el grupo que aprendió modismos también mostró una mejora en los resultados de las pruebas. Sin embargo, no se evaluó la eficacia de un posible método que combinase la enseñanza de los modismos con la enseñanza de reglas explícitas de gramática. Así, en la investigación que se describe en el presente trabajo, se quiso determinar si la combinación de la enseñanza de modismos con la enseñanza explícita de gramática puede resultar en un método aún más eficaz que una enseñanza explícita sin modismos. Es decir, ¿ayuda el aprendizaje de modismos o frases idiomáticas a la retención de los conceptos gramaticales presentados? Se puede asumir que los estudiantes a los que se les presentan modismos que ejemplifican la estructura gramatical estudiada tienen a su disposición ejemplares memorables a los que recurrir como un esquema o ayuda mnemotécnica a la hora de generar o reconocer nuevos ejemplos u oraciones. Los beneficios mnemotécnicos de los modismos pueden ayudar a la instrucción explícita de la gramática más que solo la enseñanza y práctica de unas reglas o concepto gramatical. Por último, se quiso explorar la viabilidad de incorporar la enseñanza de los modismos en el aula sin detrimento del tiempo establecido para los otros contenidos del curriculum. 


\section{4. $\quad$ El aprendizaje de ser y estar en español como segunda lengua}

En la enseñanza del español como L2 pocos temas han recibido tanta atención como los verbos copulativos ser y estar. Por ejemplo, está ampliamente demostrado que ser y estar no se adquieren simultáneamente. El orden de adquisición propuesto por VanPatten (1985, 1987) y corroborado en otros estudios con ligeras modificaciones (Geeslin, 2000; Guntermann, 1992; Ryan y Lafford, 1992) apunta a una adquisición temprana del verbo ser, que se convierte en el verbo usado "por defecto" en el habla de los aprendices de nivel inicial. La adquisición de estar ocurre gradualmente, y se utiliza con precisión primero en casos donde sólo estar es posible (como en el presente progresivo "María está/*es haciendo la tarea"). Los usos locativos y los usos copulativos con adjetivos donde también ser es posible (como ser/estar guapo/a) se incorporan más tarde, aunque existe desacuerdo en el orden específico de las etapas de adquisición. ${ }^{2}$ La Tabla 1 muestra las diferentes etapas de adquisición propuestas para los verbos copulativos (adaptada de VanPatten, 2010, p. 32).

Tabla 1. Etapas de adquisición de los verbos copulativos (VanPatten, 1987)

\begin{tabular}{|l|l|}
\hline 1. Ausencia de verbos copulativos & $\begin{array}{l}\text { Juan muy inteligente. } \\
\text { Juan no aquí. }\end{array}$ \\
\hline $\begin{array}{l}\text { 2. Adquisición y sobregeneralización de ser (incluso para } \\
\text { aquellos contextos donde se requiere el uso de estar) }\end{array}$ & $\begin{array}{l}\text { Juan es alto. } \\
\text { Juan no es aquí. } \\
\text { Juan es estudia. (= está estudiando) } \\
\text { Juan es muy contento. }\end{array}$ \\
\hline 3. Aparición de estar con el gerundio & Juan está estudiando. \\
\hline $\begin{array}{l}\text { 4. Aparición de estar para expresar localización espacial } \\
\text { y temporal }\end{array}$ & Juan no está en la cocina. \\
\hline $\begin{array}{l}\text { 5. Aparición de estar con adjetivos para expresar } \\
\text { condiciones }\end{array}$ & Juan está muy contento. \\
\hline
\end{tabular}

Aunque existan ligeras modificaciones en cuanto al orden de adquisición propuesto, lo que sí es cierto es que todos los estudios están de acuerdo en que ser siempre se adquiere antes que estar y que se generaliza su uso a contextos en los que solo estar es posible, y que el uso de estar que antes se adquiere es el que acompaña al gerundio. El uso de estar con adjetivos para expresar condiciones es uno de los que más tarde aparecen. Por ejemplo, Geeslin (2000) demostró que los estudiantes diferencian entre los dos verbos primero con respecto al criterio de la permanencia (un método de enseñanza que se encuentra a menudo en las clases de español de segunda lengua), y después con respecto a una marca de referencia (es decir, comparando el referente consigo mismo).

Por último, cabe señalar que todas estas etapas de adquisición no se recorren en poco tiempo. Los estudios que se han realizado con aprendices avanzados (como el de Guntermann, 1992) muestran que incluso a este nivel todavía no se han llegado a dominar todos los usos de ser y estar. Puesto que el presente estudio evalúa estudiantes de un nivel intermedio, no se espera que nuestros participantes hayan pasado por todas las etapas de adquisición. Dado que la adquisición y uso de los verbos copulativos ser y estar son centrales en el desarrollo de la competencia lingüística en español como L2 y que conlleva un proceso lento de aprendizaje, es importante establecer métodos eficaces para su enseñanza. El estudio que aquí se describe hace un seguimiento del realizado por Zyzik y Marqués-Pascual (en prensa) en el que se investiga el uso de los modismos para promover el aprendizaje incidental de ser y estar. De manera complementaria aquí proponemos el aprendizaje explícito de los verbos copulativos, en un acercamiento que incorpora modismos y expresiones idiomáticas como herramienta pedagógica. 


\section{Descripción del estudio}

Según los objetivos generales mencionados, se formularon las siguientes preguntas de investigación:

1. ¿Es el aprendizaje de modismos, en combinación con la enseñanza explícita de la gramática (en este caso ser/estar), más eficaz que el uso de otro tipo de actividades?

2. ¿Ayuda el aprendizaje de modismos como recurso mnemotécnico a la ejecución de reglas de gramática?

\subsection{Participantes y grupos}

El estudio se llevó a cabo con 52 estudiantes de español de nivel intermedio matriculados en una universidad pública de los Estados Unidos. La media de edad de los participantes era de 20 años, con un rango de edad que variaba entre 18 y 29 años. La mayoría de los participantes eran hablantes nativos del inglés, excepto un participante cuya lengua nativa era el japonés. Se eliminó del estudio a dos participantes cuya lengua nativa era el español o el portugués (este último por su similitud con el español con respecto a los verbos copulativos).

Los 52 participantes provenían de tres clases diferentes del mismo nivel (quinto trimestre de español como lengua extranjera). Una clase sirvió como grupo de control ( $n=$ 17) y las otras dos clases constituyeron los grupos experimentales $(n=19$ y $n=16)$. Cabe destacar que los grupos no se asignaron al azar y por lo tanto se trata de una investigación cuasiexperimental. Los dos grupos experimentales recibieron instrucción explícita mediante la presentación deductiva de las reglas de uso de ser y estar, pero con una diferencia notable entre ellos: un grupo practicó las reglas con una serie de actividades de reconocimiento y producción (input/output), mientras que el otro grupo realizó una serie de actividades con modismos que incluían los verbos ser y estar y que ejemplificaban las reglas presentadas. La Tabla 2 resume los grupos y tratamientos del estudio. En el Apéndice A se incluye la lista final de los modismos utilizados en este estudio.

Tabla 2. Grupos y tratamientos

\begin{tabular}{|c|c|c|}
\hline $\begin{array}{c}\text { Grupo de práctica gramatical } \\
(\mathrm{n}=19)\end{array}$ & $\begin{array}{c}\text { Grupo de modismos } \\
(\mathrm{n}=16)\end{array}$ & $\begin{array}{c}\text { Grupo de control } \\
(\mathrm{n}=17)\end{array}$ \\
\hline Reglas + actividades de input/output & Reglas + actividades con modismos & Control \\
\hline
\end{tabular}

\subsection{Procedimiento e instrumentos}

Para medir la incidencia de los dos acercamientos pedagógicos, se realizó un diseño de pretest-posttest con el grupo de control y los dos grupos experimentales. Durante la tercera semana del trimestre académico todos los participantes realizaron una prueba de evaluación antes de comenzar la fase de instrucción y contestaron una breve encuesta personal que contenía preguntas sobre su experiencia con el español. Durante la cuarta y la quinta semana se llevó a cabo el tratamiento pedagógico con los grupos experimentales. Durante la quinta semana los tres grupos realizaron la misma prueba de evaluación y además los dos grupos experimentales completaron un cuestionario adicional que sirvió para averiguar si los participantes recordaban las reglas presentadas. Por último, el grupo que realizó actividades con modismos también realizó una prueba de reconocimiento de los modismos aprendidos. 
A continuación examinamos detalladamente la prueba de evaluación: una de producción escrita de descripción de fotos. Esta prueba es la misma que se usó en el estudio de Zyzik y Marqués-Pascual (2012), y consistió en una presentación PowerPoint con 24 fotos. Los participantes tenían que escribir una oración para cada foto. Así, la prueba constaba de 24 ítems, de los cuales 8 requerían el uso de ser y 8 requerían el uso de estar. Dentro de cada categoría de ser y estar había a su vez una división por tipo de colocación siguiendo las reglas presentadas: ser + SN (4), ser + SAdj (4), estar + SAdj (4), estar + SPrep (4). Los 8 ítems restantes sirvieron como distractores e incluían oraciones con verbos transitivos. Para restringir el número de posibles oraciones que los participantes podían escribir para describir las fotos, al lado de cada foto aparecían unas palabras que los participantes debían usar en sus oraciones: el sujeto de la oración y un complemento. Se les indicó a los participantes que debían escribir oraciones completas y añadir otras palabras necesarias (por ejemplo, cuando los participantes vieran una foto con las palabras el estadio/lleno, debían escribir "El estadio está lleno"). Cada foto permaneció en la pantalla durante 15 segundos antes de que apareciera la siguiente foto. Los participantes escribieron las oraciones en una hoja de papel durante el tiempo en que las fotos aparecían en la pantalla.

\subsection{El tratamiento pedagógico}

Durante las semanas cuarta y quinta del trimestre los dos grupos experimentales participaron en dos sesiones de 50 minutos cada una que tuvieron lugar durante la clase a la que asistían los participantes. La primera sesión durante la semana cuarta consistió en una clase sobre el verbo ser. La segunda sesión durante la semana quinta consistió en una clase sobre el verbo estar. Como ya se ha mencionado, ambos grupos recibieron durante estas sesiones un tratamiento de enseñanza deductiva de las reglas de uso de los verbos copulativos. Esta presentación se limitó a tres reglas para cada verbo y estaban basadas en colocaciones, es decir, tomando como punto de partida el tipo de sintagma que sigue al verbo copulativo y no las diferencias semánticas que puedan existir entre ambos verbos (como la distinción tradicional de característica permanente vs. temporal). Estas reglas siguen las recomendaciones expuestas en y Marqués-Pascual y Zyzik (2012); la Tabla 3 muestra un resumen de las reglas adaptadas del inglés, lengua en la que fueron presentadas a los participantes.

Tabla 3. Reglas de uso de ser y estar basadas en colocaciones

\begin{tabular}{|l|l|l|l|}
\hline \multicolumn{2}{|c|}{ SER } & \multicolumn{2}{c|}{ ESTAR } \\
\hline $\begin{array}{l}\text { Se usa SER para unir un } \\
\text { sujeto a un sustantivo o grupo } \\
\text { nominal. }\end{array}$ & $\begin{array}{l}\text { Juan es un cero a la } \\
\text { izquierda } .\end{array}$ & $\begin{array}{l}\text { Se usa ESTAR + gerundio } \\
\text { para expresar acciones en } \\
\text { progreso. }\end{array}$ & $\begin{array}{l}\text { Juan está } \\
\text { estudiando. }\end{array}$ \\
\hline $\begin{array}{l}\text { Se usa SER con adjetivos } \\
\text { para distinguir una persona } \\
\text { o cosa de otras personas o } \\
\text { cosas. }\end{array}$ & $\begin{array}{l}\text { Juan es más listo que } \\
\text { el hambre. }\end{array}$ & $\begin{array}{l}\text { Se usa ESTAR con } \\
\text { adjetivos para describir una } \\
\text { condición que distingue el } \\
\text { estado actual de la persona } \\
\text { o cosa con otros estados de } \\
\text { sí mismo. }\end{array}$ & $\begin{array}{l}\text { Juan está más solo } \\
\text { una. }\end{array}$ \\
\hline $\begin{array}{l}\text { Se usa SER con un grupo } \\
\text { preposicional para hablar de } \\
\text { la localización y la hora de } \\
\text { eventos (no de objetos físicos } \\
\text { o personas). }\end{array}$ & $\begin{array}{l}\text { La fiesta es en casa de } \\
\text { Juan. }\end{array}$ & $\begin{array}{l}\text { Se usa ESTAR con un } \\
\text { grupo preposicional para } \\
\text { hablar de la localización o } \\
\text { condición de una persona, } \\
\text { lugar o cosa. }\end{array}$ & $\begin{array}{l}\text { La castá en el quino. } \\
\text { pinto }\end{array}$ \\
\hline
\end{tabular}


La diferencia entre los dos tratamientos se basó en el tipo de actividades que acompañaban la presentación de las reglas. El primer grupo de práctica gramatical $(n=17)$ realizó una serie de actividades de input/output propuesta por Zyzik y Marqués-Pascual (2012). Las sesiones de este grupo de práctica gramatical consistieron en los siguientes componentes: presentación de las reglas, reconocimiento del uso de las reglas en un texto y actividades de producción oral, todo ello acompañado de corrección de errores. El segundo grupo experimental ( $n=16$ estudiantes) realizó una serie de actividades de identificación del significado de modismos y su uso en contexto siguiendo las recomendaciones de los estudios de Zyzik (2010, 2011). Para cada sesión se utilizaron 18 modismos, organizados en tres grupos temáticos. Cada grupo de modismos se presentó siguiendo la siguiente secuencia: deducción del significado mediante su emparejamiento con el equivalente en inglés, relleno de huecos (sentence completion) con los modismos y sustitución de oraciones literales en contexto con un modismo (véanse ejemplos de estas actividades en el Apéndice B). Las sesiones de instrucción de los modismos siguieron la siguiente secuencia: presentación de las reglas de ser y estar, actividades con diferentes grupos de modismos y clasificación de cada grupo de acuerdo con las tres reglas presentadas. Así, se considera que cada modismo sirvió como ejemplo de una de las reglas. Finalmente, el tercer grupo ( $n=19$ estudiantes) fue un grupo de control, el cual realizó tareas de lectura previamente establecidas en el programa.

\subsection{Método de análisis de datos}

La prueba de producción escrita fue calificada siguiendo un criterio estrictamente binario: 1 punto por uso correcto de ser/estar y 0 puntos por uso incorrecto. Otro tipo de errores como errores de ortografía o morfología verbal no se tomaron en cuenta ya que no se consideraron relevantes para el estudio. El número de este tipo de errores fue muy escaso ya que los participantes tenían que usar las palabras que se les daba para construir las oraciones. Como el estudio sigue un diseño de pretest-postest fue necesario determinar si los grupos diferían entre sí al inicio del estudio. Para ello, se llevó a cabo un análisis múltiple de varianza (MANOVA); no se detectaron diferencias significativas entre los tres grupos de participantes en la prueba de producción escrita. Se asume, por lo tanto, que los tres grupos tenían un nivel comparable de conocimiento de serlestar al principio del estudio. Para medir el incremento en la prueba de evaluación se llevó a cabo un análisis de varianza de medidas repetidas (repeated measures [ANOVA]) de dos factores. El factor intragrupal fue el momento de evaluación, con dos niveles, el inicio y el final del estudio. El factor intergrupal, el grupo, consta de tres niveles: grupo de control, grupo de práctica gramatical y el grupo de modismos. En cuanto al cuestionario sobre el conocimiento de las reglas, este también se calificó de manera binaria: 1 punto por cada regla correcta y 0 puntos por cada regla incorrecta. Las respuestas parcialmente correctas (por ejemplo, cuando el ejemplo no se correspondía con la regla o era incorrecto) se contabilizaron como 0.5. El análisis estadístico de los resultados se realizó con el programa de software SPSS versión 22.

\section{Resultados}

\subsection{Prueba de producción escrita}

En primer lugar examinaremos los resultados de la prueba de producción. Recordemos que la puntuación máxima de esta prueba era 8 puntos para los ítems que requerían el uso de ser y 8 puntos para los ítems que requerían el uso de estar. Los promedios para las oraciones 
con ser fueron muy altos para todos los grupos de participantes. Hay que recordar que dentro de cada categoría con ser y con estar había en la prueba dos categorías; por tanto, se usó un test de análisis de varianza de medidas repetidas para determinar si había diferencias entre dichas categorías. Los resultados del análisis ANOVA indicaron una diferencia significativa entre las categorías, $\mathrm{F}(3,49)=25.12, \mathrm{p}<.01$. Esto significa que no todos los tipos de ítems tuvieron el mismo nivel de dificultad para los participantes. Las comparaciones posteriores entre categorías confirmaron que ser+SN resultó ser la categoría más fácil para todos los participantes en la prueba inicial, con resultados que casi rozaron la máxima puntuación (ceiling effect) y los dos tipos de ítems con estar los de mayor nivel de dificultad. La Tabla 4 muestra los resultados de todos los grupos en cuanto al tipo de ítem.

Tabla 4. Diferencia entre las categorías de la prueba inicial de producción

\begin{tabular}{|l|c|l|}
\hline Categorías & M (DS) (máx 4) & Diferencias entre las categorías \\
\hline Ser + SN & $3.90(0.35)$ & a \\
\hline Ser + Adj & $3.60(0.63)$ & a, b \\
\hline Estar + Adj & $3.44(0.80)$ & b, c \\
\hline Estar + SP & $2.94(1.14)$ & c \\
\hline
\end{tabular}

$\mathrm{M}=$ promedio, $\mathrm{DS}=$ desviación estándar

Una vez comprobado que casi todos los participantes puntuaron muy alto en los ítems con ser, pasamos a analizar los resultados con respecto a la incidencia de la enseñanza entre la prueba inicial y la final. En la Tabla 5 se presentan las estadísticas descriptivas de los resultados de la prueba de producción escrita por grupo.

Tabla 5. Resultados de la prueba de producción escrita

\begin{tabular}{|l|c|c|c|c|}
\hline & \multicolumn{2}{|c|}{ SER (máx 8) } & \multicolumn{2}{c|}{ ESTAR (máx 8) } \\
\hline Grupo & $\begin{array}{c}\text { Prueba inicial } \\
\text { M (DS) }\end{array}$ & $\begin{array}{c}\text { Prueba final } \\
\text { M (DS) }\end{array}$ & $\begin{array}{c}\text { Prueba inicial } \\
\text { M (DS) }\end{array}$ & $\begin{array}{c}\text { Prueba final } \\
\text { M (DS) }\end{array}$ \\
\hline Control $(n=17)$ & $7.71(0.47)$ & $7.76(0.43)$ & $6.82(1.17)$ & $6.76(1.64)$ \\
\hline Práctica $(n=19)$ & $7.42(0.83)$ & $7.42(0.83)$ & $\mathbf{6 . 1 6}(\mathbf{1 . 0 1})$ & $7.00(1.29)$ \\
\hline Modismos $(n=16)$ & $7.38(0.88)$ & $7.37(0.71)$ & $\mathbf{6 . 1 9}(\mathbf{1 . 6 0})$ & $\mathbf{7 . 0 6}(\mathbf{1 . 7 3})$ \\
\hline Todos $(\mathrm{n}=52)$ & $7.50(0.75)$ & $7.52(0.67)$ & $\mathbf{6 . 3 8}(\mathbf{1 . 1 8})$ & $\mathbf{6 . 9 4}(\mathbf{1 . 5 2})$ \\
\hline
\end{tabular}

$\mathrm{M}=$ promedio, $\mathrm{DS}=$ desviación estándar

Como hemos mencionado, no hubo diferencias significativas entre los grupos antes de empezar el estudio, ni en las oraciones con ser, $\mathrm{F}(2,49)=0.95, \mathrm{p}=0.39$, ni en las oraciones con estar, $\mathrm{F}(2,49)=1.50, \mathrm{p}=0.23$. Respecto a la incidencia de la enseñanza, los resultados de la ANOVA de medidas repetidas indicaron que no hubo un incremento significativo entre la prueba inicial y la prueba final con respecto al verbo $\operatorname{ser}(\mathrm{p}>.05)$. Sin embargo, con respecto al verbo estar sí hubo un claro incremento en los promedios de los dos grupos experimentales ( $\mathrm{p}=0.028$ para el grupo de práctica gramatical y $\mathrm{p}=0.039$ para el grupo de modismos), pero no para el grupo de control $(\mathrm{p}>$.05). Claramente el grupo de control se estancó en cuanto a su uso del verbo estar en las 4 semanas de diferencia entre el momento del inicio y del final del 
estudio, mientras que los dos grupos experimentales sí avanzaron en el curso de adquisición. Puesto que hay diferencias significativas entre el inicio y el final del estudio para los grupos experimentales con respecto al uso del verbo estar, se optó por investigar dónde residían las diferencias en cuanto al tipo de ítem en el caso del verbo estar. La Tabla 6 muestra los promedios de cada grupo respecto a los dos tipos de ítems.

Tabla 6. Resultados por categoría de ítems con estar en la prueba de producción escrita

\begin{tabular}{|l|c|c|c|c|}
\hline & \multicolumn{2}{|c|}{ estar + SAdj (máx 4) } & \multicolumn{2}{c|}{ estar + SPrep (máx 4) } \\
\hline Grupo & $\begin{array}{c}\text { Prueba inicial } \\
\text { M (DS) }\end{array}$ & $\begin{array}{c}\text { Prueba final } \\
\text { M (DS) }\end{array}$ & $\begin{array}{c}\text { Prueba inicial } \\
\text { M (DS) }\end{array}$ & $\begin{array}{c}\text { Prueba final } \\
\text { M (DS) }\end{array}$ \\
\hline Control $(\mathrm{n}=17)$ & $3.76(0.44)$ & $3.47(0.87)$ & $\mathbf{3 . 0 6}(\mathbf{1 . 2 0})$ & $\mathbf{3 . 2 9}(\mathbf{1 . 3 6})$ \\
\hline Práctica $(\mathrm{n}=19)$ & $\mathbf{3 . 3 2}(\mathbf{0 . 7 5})$ & $\mathbf{3 . 4 7}(\mathbf{0 . 8 4})$ & $\mathbf{2 . 8 4}(\mathbf{1 . 0 1})$ & $\mathbf{3 . 5 3}(\mathbf{0 . 9 0})$ \\
\hline Modismos $(\mathrm{n}=16)$ & $\mathbf{3 . 2 5}(\mathbf{1 . 0 6})$ & $\mathbf{3 . 6 9}(\mathbf{0 . 8 7})$ & $\mathbf{2 . 9 4}(\mathbf{1 . 2 9})$ & $\mathbf{3 . 3 8}(\mathbf{1 . 0 2})$ \\
\hline Total $(\mathrm{n}=52)$ & $\mathbf{3 . 4 4}(\mathbf{0 . 8 0})$ & $\mathbf{3 . 5 4}(\mathbf{0 . 8 5})$ & $\mathbf{2 . 9 4}(\mathbf{1 . 1 4})$ & $\mathbf{3 . 4 0}(\mathbf{1 . 0 8})$ \\
\hline
\end{tabular}

Examinaremos primero la incidencia de la instrucción para la categoría de estar+SAdj. Los resultados de la ANOVA de medidas repetidas indican que no hubo un cambio significativo antes y después del tratamiento $(\mathrm{p}>.05)$, pero sí una interacción significativa entre la variable tiempo (antes y después del tratamiento) y grupo $(\mathrm{p}=.039)$. Es decir, el efecto de la instrucción no fue el mismo para los tres grupos. Claramente, el grupo de control no mejoró, mientras que los dos grupos experimentales sí mejoraron, clarificando entonces que el tratamiento en este caso sí produjo un cambio significativo. Por lo tanto, ambos grupos experimentales mejoraron en su uso de estar+SAdj tras participar en este estudio. En cuanto a la categoría de estar+SPrep, sí hubo un efecto significativo entre el inicio y el final del estudio $(\mathrm{p}=.015)$, pero no una interacción significativa entre el momento de evaluación y el grupo $(\mathrm{p}>$.05). Por lo tanto, los tres grupos mejoraron en su uso de estar+SPrep entre el inicio y el final del estudio. Para aclarar si hubo diferencias entre los grupos, se realizó una serie de comparaciones utilizando la corrección de Bonferroni, pero no se detectaron diferencias significativas. O sea, ninguno de los tres grupos mejoró significativamente más que los otros.

\subsection{Cuestionarios}

Recordamos al lector que los dos grupos experimentales rellenaron al final del estudio un cuestionario sobre el conocimiento de las reglas presentadas. Se les pidió a los participantes que enunciaran las tres reglas que habían aprendido e ilustraran cada una con un ejemplo. La prueba rendía como resultado un máximo de 3 puntos para ser y 3 puntos para estar (puesto que los participantes aprendieron tres reglas). La Tabla 7 presenta los resultados de los dos grupos experimentales en cuanto a su retención de las reglas aprendidas.

Tabla 7. Conocimiento de las reglas

\begin{tabular}{|l|c|c|}
\hline Grupo & Reglas para ser (máx. 3) & Reglas para estar (máx. 3) \\
\hline Práctica $(\mathrm{n}=19)$ & $2.21(0.82)$ & $2.28(0.85)$ \\
\hline Modismos $(\mathrm{n}=17)$ & $0.84(1.02)$ & $1.28(1.03)$ \\
\hline
\end{tabular}


Como se puede ver en la Tabla 7, el grupo de práctica gramatical fue claramente superior a la hora de poder enunciar las reglas (por lo cual asumimos que los participantes de dicho grupo las recordaron mejor). Los resultados de un test de ANOVA indicaron que hubo una diferencia significativa entre los grupos ( $p<.01$ en ambos casos), lo cual confirma los resultados superiores del grupo de práctica gramatical. Esto parece un resultado lógico puesto que este grupo realizó varias actividades de reconocimiento y posterior producción que les obligaban a poner en práctica las reglas aprendidas (de hecho, los participantes se remitieron a la presentación de las reglas en varias ocasiones durante la lección). Sin embargo, cabe resaltar que el poder recordar y enunciar las reglas no parece haber ayudado especialmente al grupo de práctica gramatical, puesto que el grupo de modismos obtiene un desempeño equivalente o mejor en la prueba de producción (véase la Tabla 6). De hecho, para determinar si existía una relación entre el conocimiento de las reglas gramaticales y la habilidad de usar ser y estar correctamente en la prueba de producción se realizaron una serie de tests de Correlación Pearson. Los resultados de estos tests indicaron que en ninguno de los casos hubo una correlación significativa entre el conocimiento de las reglas por parte de los participantes y sus resultados en la prueba de producción $(\mathrm{p}>.05)$.

\section{Discusión e implicaciones pedagógicas}

Los resultados presentados se pueden resumir de la siguiente manera. En primer lugar, el uso de ser con sintagmas nominales $(s e r+\mathrm{SN})$ y con sintagmas adjetivales (ser+SAdj) parece ya haber sido adquirido entre estos aprendices de nivel intermedio. Recordemos que ser es el verbo que antes se adquiere y es usado "por defecto", incluso en contextos donde se debe usar estar. Por lo tanto, es de esperar que la puntuación en los ítems con ser roce la máxima posible. El hecho de que los resultados no mostraran incidencia del tratamiento con respecto al uso de ser sugiere que los aprendices podrían haber aprovechado este método de enseñanza incluso antes en su curso de aprendizaje.

Segundo, la enseñanza explícita de las reglas de ser y estar tiene efectos positivos en el uso de estar con sintagmas adjetivales (estar+SAdj) para expresar condiciones. Recordemos que en las etapas de adquisición propuestas por los diversos estudios comentados (Geeslin, 2000; VanPatten, 1985, 1987) la adquisición del verbo estar con adjetivos para expresar condición se considera el último uso de estar adquirido por los aprendices. Mientras que el grupo de control no mejoró en la producción de estar con sintagmas adjetivales para expresar condiciones (como en El hombre está enojado), la enseñanza explícita de estas reglas sí fue eficaz en este respecto, lo cual es un resultado alentador. Por último, cabe resaltar que en cuanto a la última categoría evaluada, estar con sintagmas preposicionales para expresar localización (como en El satélite está en el espacio) los tres grupos mejoraron entre el inicio y el final del estudio, incluyendo el grupo de control, el cual no recibió instrucción específica sobre estos verbos. Si nos remitimos de nuevo a las etapas de adquisición de los verbos copulativos, la adquisición de este uso de estar es más temprana que la de estar con adjetivos y es posible que el grupo de control también haya mejorado al final del estudio como resultado natural de su curso de aprendizaje al continuar sus estudios de esta lengua. Es importante destacar, sin embargo, que si observamos los promedios obtenidos por los tres grupos (véase la Tabla 6) los dos grupos experimentales obtuvieron mejores resultados que el grupo de control, si bien la 
diferencia entre los tres grupos no resultó significativa. Por último, el hecho de que los grupos experimentales superaran al grupo de control en el uso de estar confirma que los estudiantes siguen necesitando instrucción en el nivel intermedio.

De estos resultados se derivan importantes implicaciones pedagógicas. Primero, como aboga VanPatten (2010) hay que hacer un esfuerzo para que los materiales didácticos estén guiados por los resultados de las investigaciones sobre los procesos de adquisición de segundas lenguas. Sirva como ejemplo la presentación de los verbos copulativos en la mayoría de los libros de texto, donde se hace una lista exhaustiva de los diferentes usos, mediante un análisis contrastivo que puede abarcar desde nociones semánticas (como que los adjetivos pueden cambiar de significado según el verbo que se use, citando equivalentes en inglés como estar aburrido "to be bored" vs. ser aburrido "to be boring"), pasando por criterios sintácticos (por ejemplo, "ser se usa con la voz pasiva"), o ejemplos de colocaciones habituales (estar a dieta, estar a favor, estar de moda, etc.). La mayoría de estas listas exhaustivas con una serie de usos para ser y una serie de usos para estar, se presentan, además, en una sola lección o secuencia de lecciones. Este tipo de acercamiento pedagógico no sigue lo que se ha demostrado en cuanto al orden de adquisición de los verbos copulativos. Podríamos así proponer un acercamiento pedagógico en que la instrucción explícita enfatice cuándo y en qué contextos hay que usar estar y no tanto ser, introduciendo los usos en etapas que reflejen el curso natural de adquisición: el uso de estar con el presente progresivo, seguido del uso de estar con sintagmas preposicionales (tanto para expresar condición -como en estar a dieta-, como para expresar ubicación) y por último el uso de estar con adjetivos de condición. En sus principios para el aprendizaje de segundas lenguas en contextos de instrucción formal, Ellis (2005) afirma que es preciso que la enseñanza siga el "programa interno" de los aprendices ("Instruction needs to take into account the learner built-in syllabus", p. 216). Los resultados de este estudio confirman que se puede trabajar en consonancia con ese programa interno para facilitar o incluso agilizar el avance en el curso de aprendizaje, sobre todo hacia aquellas etapas que ofrezcan más dificultad.

Volviendo a la primera pregunta de investigación, no podemos concluir que la instrucción explícita de reglas gramaticales en combinación con el aprendizaje de modismos sea más eficaz que la instrucción explícita con otro tipo de actividades. Ambos grupos experimentales aprovecharon el tratamiento pedagógico y mejoraron en su uso de estar con adjetivos y con sintagmas preposicionales sin diferencias significativas entre ellos. Sin embargo, nuestros resultados indican que la práctica de reglas gramaticales con modismos es una opción de enseñanza perfectamente viable y que no hay razón para renunciar a la enseñanza directa de modismos en la clase, sobre todo si se puede "matar dos pájaros de un tiro": los estudiantes en nuestro grupo experimental no solo mejoraron en su habilidad de uso de ser y estar, sino que además aprendieron una serie de modismos que ya no les van a resultar completamente desconocidos en el futuro. Como Zyzik (2010), consideramos esto un punto de partida que le puede permitir al aprendiz seguir ampliando su conocimiento del lenguaje idiomático en el futuro.

En cuanto a nuestra segunda pregunta, no parece que los modismos funcionaran como ejemplos mnemotécnicos a la hora de recordar las reglas aprendidas. Sin embargo, sí sirvieron como herramienta pedagógica a la hora de usar y producir la estructura gramatical. Recordemos que los participantes que más practicaron las reglas en las actividades demostraron recordar las reglas mejor que el grupo que practicó modismos. Sin embargo, el grupo de modismos, pese a no recordar las reglas, obtuvo resultados equivalentes a la hora de usar los verbos copulativos en 
la prueba final. Se puede argumentar que lo que les sirvió a estos estudiantes fue la exposición a múltiples y numerosos ejemplos de las colocaciones evaluadas, lo cual pudo reforzar el aprendizaje incidental de dichas reglas y ayudar en su posterior aplicación. Coincidimos aquí con las conclusiones de Zyzik y Marqués-Pascual (2012) con respecto a los efectos de frecuencias en el input: se puede cuestionar la importancia de presentar a los aprendices unas reglas o de buscar la mejor manera de explicar un concepto gramatical, para dar más importancia al tipo de input y contacto con la lengua que se le proporciona a los estudiantes.

\section{Conclusión}

Con este estudio empírico no se pretendía resolver cuál es la mejor manera de incorporar la enseñanza de modismos, ni tampoco tratar la importancia de abordar cuestiones pragmáticas y culturales del lenguaje idiomático en el aula de segunda lengua. Se propuso investigar la viabilidad de incorporar modismos en el aula de lengua como herramienta pedagógica para reforzar el aprendizaje de la gramática. La enseñanza de modismos no fue más eficaz que el uso de otro tipo de actividades de reconocimiento en contexto y producción, y se constató que los modismos no sirvieron como elementos mnemotécnicos que favorecieran el conocimiento de las reglas de uso de ser y estar. Sin embargo, el conocimiento superior de las reglas por parte de uno de los grupos experimentales no contribuyó de manera significativa a su ejecución de las mismas en una prueba de producción escrita.

La incorporación del lenguaje idiomático en la enseñanza de conceptos o estructuras gramaticales puede ser un desafío para los docentes en términos del tiempo que requiere tanto la selección de modismos adecuados que ejemplifiquen la estructura en cuestión, como la creación de materiales didácticos. Sin embargo, el uso de modismos en la enseñanza explícita de conceptos gramaticales es una práctica viable a la vez que motivadora para los estudiantes, puesto que favorece la interacción con hablantes nativos y resulta en una competencia y producción lingüística más cercana a la de estos.

\section{Notas}

1. Ellis et ál. (2008), por ejemplo, cita numerosos estudios que sugieren que el lenguaje formulaico o fraseológico (del cual el lenguaje idiomático forma un subgrupo) constituye hasta un 50\% o incluso más de la lengua hablada.

2. Se recomienda al lector interesado la panorámica expuesta por Geeslin (2014) en cuanto al orden de adquisición de los verbos ser y estar en español como L2.

\section{Bibliografía}

Boers, F. (2000). Metaphor awareness and vocabulary retention. Applied Linguistics. 21 (4), 553-571.

Boers, F., Eyckmans, J. y Stengers, H. (2007). Presenting figurative idioms with a touch of etymology: More than mere mnemonics? Language Teaching Research. 11 (1), 43-62.

Boers, F., Lindstromberg, S., y Webb, S. (2014). Further evidence of the comparative memorability of alliterative expressions in second language learning. RELC Journal. 45 (1), 85-99.

Cooper, T. C. (1998). Teaching Idioms. Foreign Language Annals. 31, 255-266. 
Cooper, T. C. (1999). Processing of idioms by L2 learners of english. TESOL Quarterly. 33 (2), 233-262.

Cornell, A. (1999). Idioms: An approach to identifying major pitfalls for learners. IRAL. 37 (1), 1-22.

Ellis, N. C., Simpson-Vlach, R., y Maynard, C. (2008). Formulaic language in native and second language speakers: Psycholinguistics, corpus linguistics, and TESOL. TESOL Quarterly. 42 (3), 375-396.

Ellis, R. (2005). Principles of Instructed Language Learning. System. 33, 209-234.

Geeslin, K. (2000). A New Approach to the Second Language Acquisition of Copula Choice in Spanish. Por R. Leow y C. Sanz. (Eds.). Spanish Applied Linguistics at the Turn of the Millennium: Papers from the 1999 Conference on the L1 \& L2 Acquisition of Spanish and Portuguese. (50-66). Somerville, MA: Cascadilla Press.

Geeslin, K. L. (2014). The Acquisition of the Copula Contrast in Second Language Spanish. Por K. L. Geeslin (Ed.). The Handbook of Spanish Second Language Acquisition. (219-234). West Sussex, UK: Wiley-Blackwell.

Guntermann, G. (1992). An Analysis of Interlanguage Development over Time: Part II, Ser and Estar. Hispania. 75, 1294-1303.

Irujo, S. (1986a). Don't put your leg in your mouth: Transfer in the acquisition of idioms in a second language. TESOL Quarterly. 20 (2), 287-304.

Irujo, S. (1986b). A piece of cake: Learning and teaching idioms. English Language Teaching Journal. 40 (3), 236-242.

Lindstromberg, S. y Boers, F. (2008). The mnemonic effect of noticing alliteration in lexical chunks. Applied Linguistics. 29 (2), 200-222.

Liontas, J. I. (1999). Developing a pragmatic methodology of idiomaticity: The comprehension and interpretation of SL vivid phrasal idioms during reading. (Tesis doctoral). University of Arizona. http://arizona.openrepository.com/arizona/handle/10150/284736 [Consulta 28 de abril de 2015].

Liontas, J. I. (2003). Killing two birds with one stone: Understanding spanish VP idioms in and out of context. Hispania. 86 (2), 289-301.

Marqués-Pascual, L. y Zyzik, E. (2012). Implicit and explicit instruction and the acquisitions of ser and estar. Ponencia presentada en American Council of Teachers of Foreign Languages, Annual Convention. Philadelphia (PA), EE.UU.

Ryan, J., y Lafford, B. (1992). Acquisition of Lexical Meaning in a Study Abroad Environment: Ser and Estar and the Granada Experience. Hispania. 75, 714-722.

Sugano, M.Z. (1981). The idiom in spanish language teaching. The Modern Language Journal. 65 (1), 59-66.

Tran, H. Q. (2012). An explorative study of idiom teaching for pre-service teachers of english. English Language Teaching. 5 (12), 76-86.

VanPatten, B. (1985). The Acquisition of Ser and Estar in Adult Second Language Learners: A Preliminary Investigation of Transitional Stages of Competence. Hispania. 68, 399-406. 
VanPatten, B. (1987). Classroom Learners' Acquisition of Ser and Estar: Accounting for Developmental Patterns. Por B. VanPatten, T. Dvorak y J. Lee (Eds.). Foreign Language Learning: A Research Perspective. (61-75). Rowley, MA: Newbury House.

VanPatten, B. (2010). Some Verbs Are More Perfect than Others: Why Learners Have Difficulty with Ser and Estar and What It Means for Instruction. Hispania: A Journal Devoted to the Teaching of Spanish and Portuguese. 93 (1), 29-38.

Wray, A. (2000). Formulaic sequences in second language teaching: Principle and practice. Applied Linguistics. 21 (4), 463-489.

Zyzik, E. (2010). Sin pelos en la lengua: La adquisición de modismos en una clase de español como lengua extranjera. Hispania. 93 (3), 453-470.

Zyzik, E. (2011). Second language idiom learning: The effects of lexical knowledge and pedagogical sequencing. Language Teaching Research. 15 (4), 413-433.

Zyzik, E., y Marqués-Pascual, L. (2012). Spanish differential object marking: An empirical study of implicit and explicit instruction. Studies in Hispanic and Lusophone Linguistics. 5, 387-421.

Zyzik, E. y Marqués-Pascual, L. (en prensa). Practice with formulaic sequences: Can it promote the incidental learning of grammar? Por C. Jones (Ed.). Practice in Second Language Learning. Cambridge: Cambridge University Press.

\section{Apéndices}

\section{Apéndice A: Modismos con ser y estar}

1. estar en las nubes

2. estar de mala leche

3. estar patas arriba

4. estar sin blanca

5. estar más solo que la una

6. estar entre la espada y la pared

7. estar por los suelos

8. estar como una cabra

9. estar al corriente

10. estar hecho polvo

11. estar en pañales

12. estar pedo

13. estar como pez en el agua

14. estar más claro que el agua

15. estar en el quinto pino

16. estar crudo

17. estar hasta el gorro

18. estar con la soga al cuello 
19. ser un don nadie

20. ser un cero a la izquierda

21. ser pan comido

22. ser más largo que un día sin pan

23. no ser nada del otro mundo

24. ser más raro que un perro verde

25. ser un arma de doble filo

26. ser más listo que el hambre

27. ser un perro faldero

28. ser una lata

29. ser un libro abierto

30. ser muy suyo

31. ser un pez gordo

32. ser un viejo verde

33. ser duro de mollera

34. ser más lento que una tortuga

35. ser (un/a) aguafiestas

36. ser la media naranja 
